\title{
Hypersensitivity Reactions To Chemotherapy: an EAACI Position Paper
}

Mauro Pagani ${ }^{1}$, Sevim Bavbek ${ }^{2}$, Emilio Alvarez-Cuesta ${ }^{3}$, patrizia Bonadonna ${ }^{4}$, Josefina Cernadas $^{5}$, Anca Chiriac ${ }^{6}$, Adile Berna Dursun ${ }^{7}$, Sahar Hamadi ${ }^{8}$, Ricardo Madrigal-Burgaleta ${ }^{9}$, Soledad Sanchez-Sanchez ${ }^{10}$, and Mariana Castells ${ }^{11}$

${ }^{1}$ ASST Mantova

${ }^{2}$ Ankara University Division of Immunology and Allergy

${ }^{3}$ Hospital Ramón y Cajal

${ }^{4}$ Azienda Ospedaliera Universitaria Integrata Verona

${ }^{5}$ University Hospital S João, Porto , Portugal

${ }^{6}$ Hôpital Arnaud de Villeneuve, University Hospital of Montpellier

${ }^{7}$ Recep Tayyip Erdoğan University

${ }^{8}$ Massachusetts General Hospital Division of Rheumatology Allergy and Immunology

${ }^{9}$ St Bartholomew's Hospital, Barts Health NHS Trust

${ }^{10}$ Allergy Department, University Hospital Complex A Coruña (CHUAC),

${ }^{11}$ Brigham and Women's Hospital

April 1, 2021

\begin{abstract}
Chemotherapeutic drugs have been widely used in the treatment of cancer disease for about 70 years and, even with the development of new therapies, they are still prescribed by oncologists, alone or in combination with other antineoplastic agents. All chemotherapies are able to provoke hypersensitivity reactions, even with different incidences, depending on the different class of these drugs, and these reactions are the third leading cause of fatal drug-induced anaphylaxis in the United States. In Europe deaths related to chemotherapy have also been reported. In particular, most reactions are provoked by platinum compounds, taxanes, epipodophyllotoxins and asparaginase. However, currently there are different points of view about the best procedures for the diagnosis, treatment and prevention of these reactions. Thus, the European Network on Drug Allergy and Drug Allergy Interest Group of the European Academy of Allergy and Clinical Immunology organized a task force to provide data and recommendations regarding the allergological work up in this field of drug hypersensitivity reactions. The aims of this position paper were to provide consensus on the investigation of HSRs to chemotherapeutic drugs and to give practical suggestions for clinicians that treat these patients, such as oncologists, allergologists and internists. Key sections cover: risk factors, pathogenesis, symptoms, role of skin tests, in vitro tests, indications and contraindications of drug provocation tests and desensitization of neoplastic patients with allergic reactions to chemotherapeutic drugs. Statements, recommendations and unmet needs were discussed and proposed at the end of each section.
\end{abstract}

\section{Hosted file}

Hypersensitivity Reactions To Chemotherapy.pdf available at https://authorea.com/users/ 405077/articles/516246-hypersensitivity-reactions-to-chemotherapy-an-eaaci-positionpaper 